\title{
Pumping and electron-stimulated desorption properties of a dual-layer nonevaporable getter
}

\author{
O.B. Malyshev, R. Valizadeh and A.N. Hannah
}

\section{Published version information:}

Citation: OB Malyshev, R Valizadeh and AN Hannah. "Pumping and electronstimulated desorption properties of a dual-layer nonevaporable getter". Journal of Vacuum Science and Technology A, vol. 34, no. 6 (2016): 061602.

doi: $\underline{10.1116 / 1.4964612}$

This article may be downloaded for personal use only. Any other use requires prior permission of the author and AIP Publishing.

This version is made available in accordance with publisher policies. Please cite only the published version using the reference above. 


\title{
Pumping and electron-stimulated desorption properties of a dual-layer nonevaporable getter
}

\author{
Oleg B. Malyshev, ${ }^{\text {a) }}$ Reza Valizadeh, and Adrian N. Hannah \\ ASTeC, STFC Daresbury Laboratory, Warrington, Cheshire WA4 4AD, United Kingdom
}

(Received 20 May 2016; accepted 23 September 2016; published 10 October 2016)

\begin{abstract}
The ASTeC Vacuum Science Group has an ongoing study for the improvement of the nonevaporable getter (NEG) coatings currently used in many accelerators around the world. The main advantages of using NEG coatings are evenly distributed pumping speed, low thermal outgassing rates, and low photon-stimulated gas desorption and electron-stimulated gas desorption (ESD). Previously, it was shown that the dense NEG coating provides lower ESD compared to that of a columnar film, but its pumping properties are reduced. This paper describes the results for a dual layer of NEG where a dense layer was deposited first and a columnar layer was then deposited on top. In such a dual-layer NEG coating, the dense layer acted as a barrier for hydrogen diffusion and the columnar layer further reduced the ESD of hydrogen and provided improved pumping properties. An alloy target of Ti-Zr-Hf-V was used to deposit a $0.5 \mu$ m-thick layer of the dense NEG followed by a $1 \mu \mathrm{m}$-thick columnar structure of NEG onto the inner surface of a 50 -cm long stainless steel tube. The composition and the structure of the dual layer were determined by energy dispersive x-ray spectroscopy and scanning electron microscopy, respectively. The NEG vacuum performance was tested using the ESD and pumping properties, which were measured on a dedicated in-house designed facility. (C) 2016 American Vacuum Society.

[http://dx.doi.org/10.1116/1.4964612]
\end{abstract}

\section{INTRODUCTION}

Since their invention in $1990 \mathrm{~s},{ }^{1-3}$ nonevaporable getter (NEG) coatings have become an important vacuum technology used in particle accelerators. ${ }^{4-7}$ The ultrahigh vacuum (UHV) and extreme high vacuum conditions along the beam vacuum chamber are specified by a tolerable beam-gas interaction rate. These conditions can be met due to the main NEG coating properties: distributed pumping speed ${ }^{1-3,8}$ as well as low thermal outgassing rates $^{2,3}$ and particleinduced $^{9-11}$ desorption yield compared to uncoated typical technical metals used for vacuum chamber. In many cases, these properties provide the only solution in a conductancelimited environment. ${ }^{4}$ It is also the most cost effective method for achieving the specified gas density. ${ }^{7,12}$

The gas density of $\mathrm{H}_{2}, \mathrm{CO}$, and $\mathrm{CO}_{2}$ along an accelerator vacuum chamber coated with $\mathrm{NEG}$ is proportional to the ratio of the desorption yield $\eta$ and the sticking probability $\alpha$ for these gases: $n \propto \eta / \alpha{ }^{13,14}$ Previous studies have determined that the columnar structure of the NEG film provides a greater sticking probability and capacity than a dense NEG film. ${ }^{8,15,16}$ The highest pumping properties were obtained with a quaternary Ti-Zr-Hf-V film with a columnar structure deposited from an alloy target. ${ }^{17}$ However, the lowest electron-stimulated gas desorption (ESD) yields were achieved with a quaternary Ti-Zr-Hf-V film with a dense structure deposited from an alloy target. ${ }^{18,19}$ This is due to the nature of the dense film as a superior barrier for hydrogen diffusion from the vacuum chamber material into the vacuum. By combining the low ESD yield of the dense film and the highest pumping

${ }^{\text {a)} E l e c t r o n i c ~ m a i l: ~ o l e g . m a l y s h e v @ s t f c . a c . u k ~}$ properties of the columnar film, one may be able to achieve an even lower gas density.

\section{EXPERIMENT}

\section{A. Sample deposition and surface analysis}

A dual layer of NEG was deposited on an internal surface of a $0.5-\mathrm{m}$ long and $38-\mathrm{mm}$ diameter $316 \mathrm{LN}$ stainless steel tube. A 3-mm diameter alloy target of Ti-Zr-Hf-V comprising equal proportions of 25 at. \% for each element was used to deposit $0.5 \mu \mathrm{m}$ of dense NEG, followed by $1 \mu \mathrm{m}$ of columnar structure, as determined with a scanning electron microscope (SEM) and shown in Fig. 1. The depositions were performed in the solenoidal magnetron deposition facility, as described in detail in Ref. 16. The depositions were carried out under the same conditions as used in our previous experiments: ${ }^{18}$ a partial pressure of $10^{-2}$ mbar of research-grade $\mathrm{Kr}$ gas, a deposition temperature of $110^{\circ} \mathrm{C}$, and using a DC and a pulsed DC mode to produce the columnar and the dense thin film structures, respectively. The tube was baked prior to deposition, and the base pressure was $10^{-10}$ mbar. Two test pieces of Si and stainless steel were deposited upon simultaneously, and were used to determine the film morphology and structure. The composition and the structure of the dual layer were determined by energy dispersive $\mathrm{x}$-ray spectroscopy, which confirmed that the deposited film possessed the same stoichiometric composition as the target.

\section{B. NEG evaluation setup and formulae}

The NEG ESD and pumping properties were measured on a dedicated in-house designed facility shown in Fig. 2. The details of the facility design and a model employed for the ESD yields calculations are described in detail in Ref. 20, 


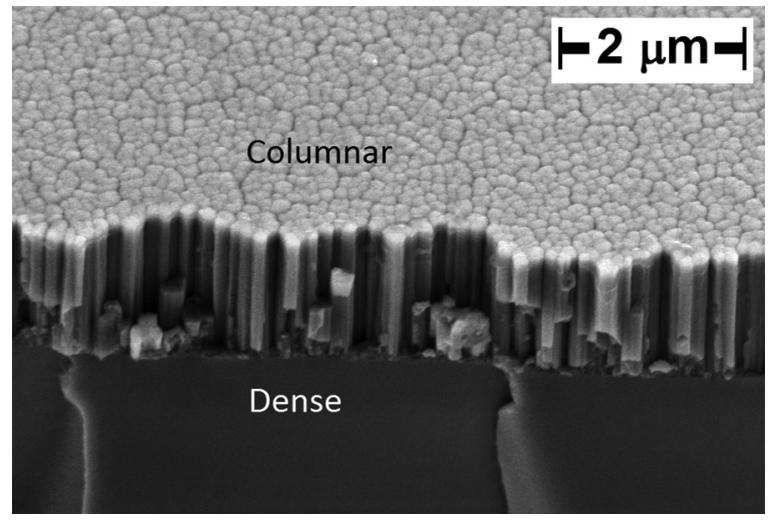

FIG. 1. Cross-section SEM micrograph of a dual-layer film.

and a method and the modeling results for the sticking probability calculations are described in Ref. 21. Pressures $P_{1}$ and $P_{2}$ are measured with residual gas analyzers (RGA) in the test chamber and used for the ESD yields and sticking probability calculations. The gas injection line is used for a selected gas injection for the sticking probability and pumping capacity measurements. Sticking probability is estimated from the pressure ratio and $P_{2} / P_{1}$ using the results from test particle Monte-Carlo modeling. ${ }^{21}$ The amount of injected gas and injected gas flow are calculated from the measured pressure and known volume in the injection line. The pumping line is used for pumping the test chamber during initial pumping down and bakeout/activation procedure (the

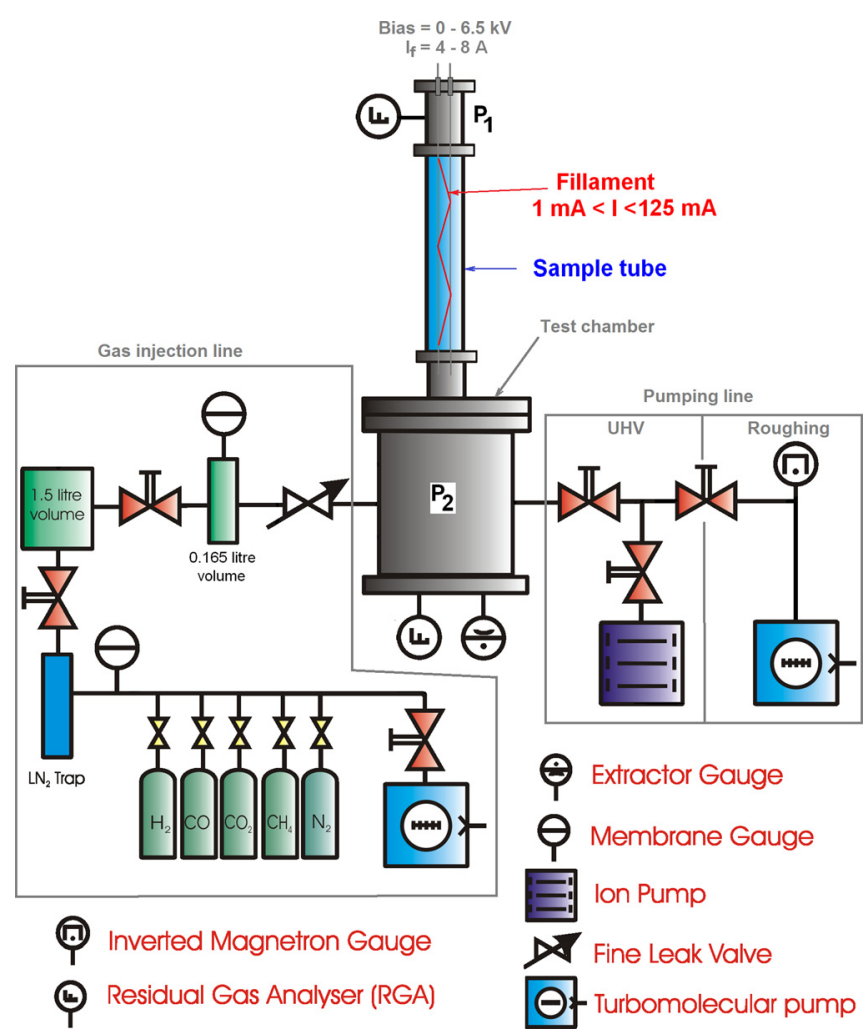

FIG. 2. (Color online) Layout of the facility for NEG ESD and pumping property measurements on tubular samples. Turbomolecular pumps are backed with scroll pumps (not shown). roughing pumping line) and during ESD measurements (the UHV pumping line).

The ESD yields are calculated with two formulas. The ESD yields for gases which are not pumped by NEG coating (such as $\mathrm{CH}_{4}$ and noble gases) are calculated with

$$
\eta_{M}=\left[\Delta P_{1, M}-\Delta P_{2, M}\right] \frac{2 U_{M} q_{e}}{k_{B} T I},
$$

and the ESD yields for gases which are pumped by NEG coating (such as $\mathrm{H}_{2}, \mathrm{CO}, \mathrm{CO}_{2}$, and $\mathrm{H}_{2} \mathrm{O}$ ) are calculated with

$$
\eta_{M}=\left[\Delta P_{1, M}+\frac{\Delta P_{1, M}-\Delta P_{2, M}}{\cosh \sqrt{\frac{\alpha_{M} C_{M}}{U_{M}}-1}}\right] \frac{\alpha_{M} C_{M} q_{e}}{k_{B} T I},
$$

where $\Delta P$ [mbar] is the pressure difference measured during and before electron bombardment, $U\left[\mathrm{~m}^{3} / \mathrm{s}\right]$ is the sample tube conductance, $\alpha_{M}$ is the sample tube wall (NEG coating) sticking probability, $C_{M}\left[\mathrm{~m}^{3} / \mathrm{s}\right]=\pi d L v_{M} / 4$ is ideal sample wall pumping speed, where $d$ and $L$ are an inner diameter and an axial length of the sample tube and $v_{M}$ is a mean velocity of gas $M$ molecules.

\section{Experimental procedure for vacuum properties evaluation}

The produced tubular sample with $1.5 \mu \mathrm{m}$ thick dual NEG layer was moved to the NEG ESD and pumping properties facility for vacuum properties evaluation. The same sample was used for all measurements described in this paper. The experimental procedure includes the following steps:

(1) Sample installation (run 1) or air vent (runs 6 and 11) followed by pumping down below $10^{-6}$ mbar.

(2) Vacuum system bakeout and/or NEG coating activation:

- For runs 1, 6, and 11 only: Vacuum system bakeout to $250{ }^{\circ} \mathrm{C}$ for $24 \mathrm{~h}$ (with a ramp-up and -down rate of $50{ }^{\circ} \mathrm{C} / \mathrm{h}$ ) after installing the sample or venting it to air. During this bakeout, the NEG-coated sample was ramped up together with the vacuum system and kept at $80^{\circ} \mathrm{C}$ for $31 \mathrm{~h}$.

- In run 10 this step was omitted.

- For other runs: The NEG activation to a selected temperature $\left(150,180,250\right.$, and $350{ }^{\circ} \mathrm{C}$ for $24 \mathrm{~h}$ with a ramp-up and -down rate of $50^{\circ} \mathrm{C} / \mathrm{h}$ ).

(3) The ESD measurement using electrons at energy of $500 \mathrm{eV}$.

(4) The $\mathrm{H}_{2}$ initial sticking probability measurement and the $\mathrm{CO}$ initial sticking probability and pumping capacity measurement followed by a full saturation of the NEG coating with $\mathrm{CO}$.

(5) After runs 5 and 10, starting from item (1). For other runs, starting from item (2).

The vacuum system was vented to air for 5 min between runs 5 and 6 and runs 10 and 11 to determine whether this was sufficient to bring the sample to its initial condition. This procedure was repeated twice (see Table I). 
TABLE I. Sticking probabilities for different gases and the CO sorption capacities for the samples activated at different temperatures, $T_{a}$.

\begin{tabular}{|c|c|c|c|c|c|c|}
\hline \multirow[b]{2}{*}{ Run no } & \multirow[b]{2}{*}{ Initial condition } & \multirow[b]{2}{*}{$T_{a}\left({ }^{\circ} \mathrm{C}\right)$} & \multicolumn{3}{|c|}{ Sticking probability } & \multirow[b]{2}{*}{ CO sticking capacity (ml) } \\
\hline & & & $\mathrm{H}_{2}$ & $\mathrm{CO}$ & $\mathrm{CO}_{2}$ & \\
\hline 1 & As-received & 80 & $1.5 \times 10^{-3}$ & $1.1 \times 10^{-2}$ & $4.5 \times 10^{-2}$ & - \\
\hline 2 & $\mathrm{CO}$ saturated & 150 & $9 \times 10^{-3}$ & $5.5 \times 10^{-2}$ & 0.1 & 0.3 \\
\hline 3 & CO saturated & 180 & $1.8 \times 10^{-2}$ & $7.5 \times 10^{-2}$ & 0.11 & 0.5 \\
\hline 4 & $\mathrm{CO}$ saturated & 250 & $2.3 \times 10^{-2}$ & $8.5 \times 10^{-2}$ & 0.2 & 3 \\
\hline 5 & CO saturated & 350 & $3.0 \times 10^{-2}$ & 0.11 & 0.2 & 10 \\
\hline 6 & Vented to air & 80 & $1.5 \times 10^{-3}$ & $5 \times 10^{-3}$ & $4.5 \times 10^{-2}$ & - \\
\hline 7 & CO saturated & 150 & $1.0 \times 10^{-2}$ & $5.5 \times 10^{-2}$ & 0.1 & 0.2 \\
\hline 8 & $\mathrm{CO}$ saturated & 180 & $1.8 \times 10^{-2}$ & $7.5 \times 10^{-2}$ & 0.11 & 0.8 \\
\hline 9 & CO saturated & 250 & $2.0 \times 10^{-2}$ & 0.18 & 0.2 & 2.5 \\
\hline 10 & CO saturated & 20 & $1.2 \times 10^{-2}$ & 0.11 & 0.11 & 0.3 \\
\hline 11 & Vented to air & 80 & $1.4 \times 10^{-3}$ & $4 \times 10^{-3}$ & $4.5 \times 10^{-2}$ & - \\
\hline 12 & $\mathrm{CO}$ saturated & 150 & $1.5 \times 10^{-3}$ & $5.5 \times 10^{-2}$ & 0.1 & 0.2 \\
\hline 13 & CO saturated 1 year pumping & 180 & $5 \times 10^{-3}$ & $8.5 \times 10^{-2}$ & 0.11 & 0.8 \\
\hline 14 & CO saturated & 250 & $8 \times 10^{-3}$ & 0.14 & 0.15 & 2 \\
\hline
\end{tabular}

Run 10 aims to observe the behavior of a CO-saturated NEG coating without activation. The vacuum system was exposed to $\mathrm{CO}$ at a pressure $P=10^{-3} \mathrm{mbar}$ for $10 \mathrm{~min}$ and then was pumped down with a turbomolecular pump for $2 \mathrm{~h}$ reaching pressure of $4 \times 10^{-10}$ mbar prior to the beginning of the ESD measurements.

After run 12, the vacuum system with a sample held pumped with a sputter ion pump for a year at a pressure $\sim 10^{-10}$ mbar, after which it was activated at $180^{\circ} \mathrm{C}$ for $24 \mathrm{~h}$.

The ESD yields were measured as a function of electron dose. The results for runs $1-6$ are shown in Fig. 3. These results can be compared with our earlier results for singlelayer dense and columnar films. ${ }^{19}$ A comparison of the ESD yields as a function of electron dose for samples activated at 80,150 , and $180^{\circ} \mathrm{C}$ is shown in Fig. 4, and the sticking probabilities for $\mathrm{H}_{2}$ and $\mathrm{CO}$ and the sorption capacity for $\mathrm{CO}$ are shown in Table I.

\section{DISCUSSION}

The ESD yields of a dual-layer film in runs 2-4 shown in Fig. 3 can be compared to the single-layer results given in Fig. 3 of Ref. 18 measured at exactly the same conditions. The ESD yields for $\mathrm{H}_{2}$ and $\mathrm{CO}$ at electron dose of $10^{21}$, $10^{22}, 10^{23}$, and $10^{24} \mathrm{e}^{-} / \mathrm{m}^{2}$ are summarized in Table II. It can be seen that the ESD yields of the dual layer are either better or comparable to those of a single layer. The pumping properties for a dual-layer film are better than those for a singlelayer dense film, but are lower than or comparable to a single-layer columnar film (cf. Table I in the present work and Table 1 in Ref. 18). The latter result can be explained by the variations in pumping properties present between samples. In general, the combined vacuum performance $(\eta / \alpha)$ of a dual-layer film is better than both single-layer films, i.e., the same activation procedures for a dual-layer film will provide lower pressures under electron bombardment than a single-layer film in a wide range of electron doses from the initial dose (just after activation) to $10^{24}-10^{25} \mathrm{e}^{-} / \mathrm{m}^{2}$. Because the photon-stimulated desorption process occurs mainly in the two steps of photoelectron production and electron-stimulated desorption, a dual-layer film will be beneficial under synchrotron radiation as well.

It is also interesting to compare the results from a duallayer film with those from single-layer films deposited on vacuum-fired stainless steel vacuum chambers. ${ }^{19}$ It can be seen that a single-layer dense film on vacuum-fired stainless steel provides comparable or lower ESD yields, though its pumping properties are not as good as those of the dual-layer film. By comparing three sets of results, comprising those reported in this paper and the two sets of data in Refs. 18 and 19 , it can be concluded that if the NEG coating provides an insufficiently low pressure, then further ESD reduction could be achieved by certain procedures. These procedures are herein set out in the order of their efficiency: (1) using a dense NEG coating instead of a columnar one, ${ }^{18}$ (2) using a dual-layer NEG coating (demonstrated in this paper), (3) vacuum firing the vacuum chamber before NEG deposition, ${ }^{19}$ and (4) using a dual-layer NEG coating deposited on a vacuum-fired vacuum chamber [the latter is a logical conclusion of the previous (1)-(3) item and should be examined in the future studies].

The optimum activation temperature for the dual-layer NEG coating depends on application and should be chosen by a user based on the following considerations. The NEG activation at $150{ }^{\circ} \mathrm{C}$ in run 2 rendered the lowest value of $\eta\left(\mathrm{H}_{2}\right)$ after a dose of $10^{24} \mathrm{e}^{-} / \mathrm{m}^{2}$ when compared to runs 1 at a lower activation temperature and runs 3-5 at a higher activation temperature, though this was not true for other gas species. The NEG activation at $180^{\circ} \mathrm{C}$ in run 3 provided the lowest initial $\eta\left(\mathrm{H}_{2}\right)$ and $\eta(\mathrm{CO})$ values; but $\eta\left(\mathrm{H}_{2}\right)$ reached its lowest value at a dose of $\mathrm{D}=4 \times 10^{22} \mathrm{e}^{-} / \mathrm{m}^{2}$, and then increased until $\mathrm{D}=6 \times 10^{23} \mathrm{e}^{-} / \mathrm{m}^{2}$ and decreased again at higher doses. This kind of behavior of $\eta\left(\mathrm{H}_{2}\right)$ for 

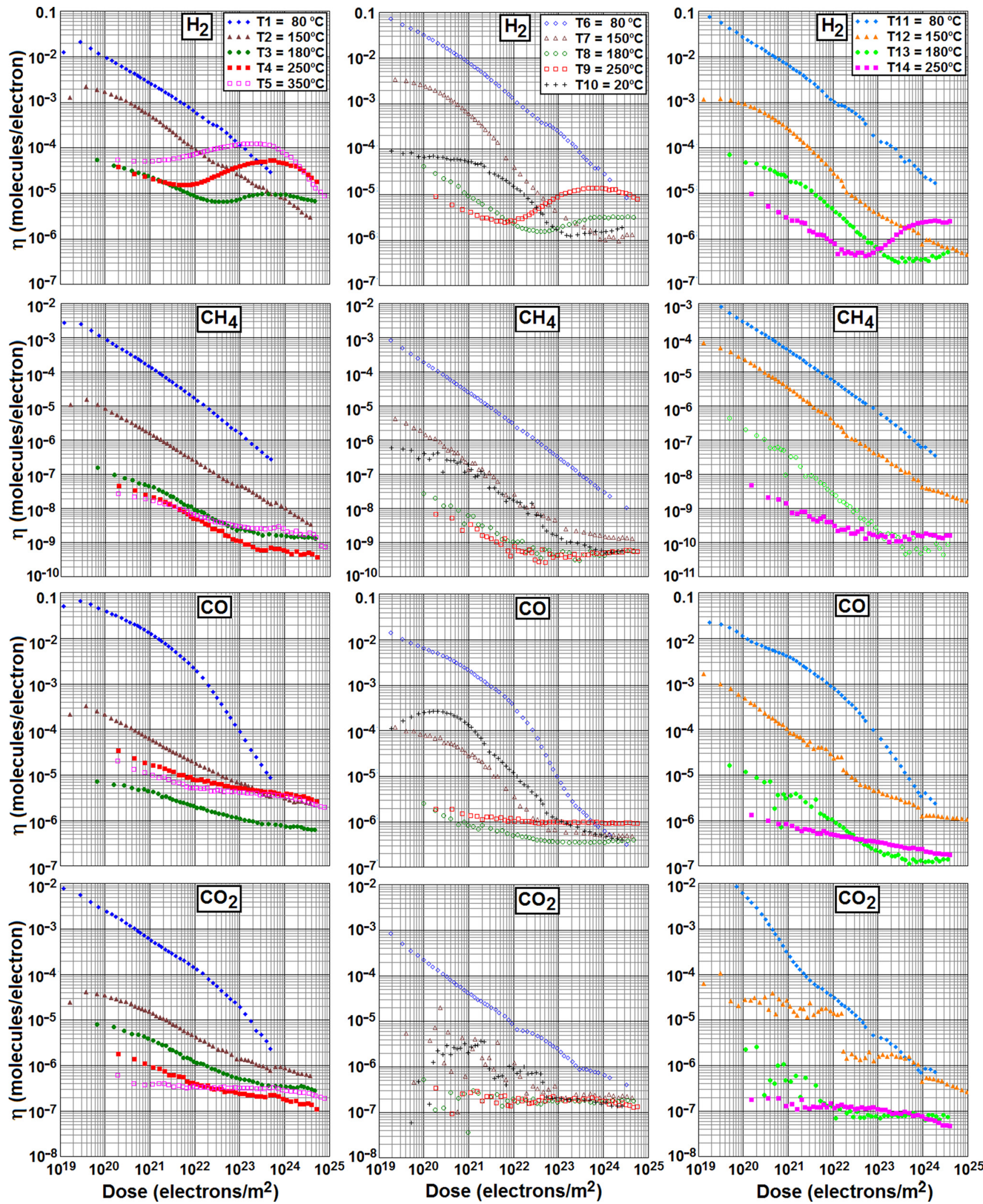

FIG. 3. (Color online) ESD yields as a function of electron dose for activated samples created by runs 1-5 (left column), runs 6-10 (middle column), and runs 11-14 (right column) for the gases (top to bottom rows) $\mathrm{H}_{2}, \mathrm{CH}_{4}, \mathrm{CO}$, and $\mathrm{CO}_{2}$. An index at $\mathrm{T}$ in the legend corresponds to the run number.

a NEG-coated vacuum chamber has been reported in Refs. 18 and 19 and is associated with hydrogen diffusion from the stainless steel substrate through the NEG coating. The higher activation temperatures $\left(250\right.$ and $\left.350^{\circ} \mathrm{C}\right)$ were not found to decrease the initial $\eta$ value for the main gases in a NEG-coated chamber $\left(\mathrm{H}_{2}, \mathrm{CH}_{4}\right.$, and $\left.\mathrm{CO}\right)$, while the $\eta\left(\mathrm{H}_{2}\right)$ increase was found to be higher and to begin at lower doses, which is also similar to the results reported in 

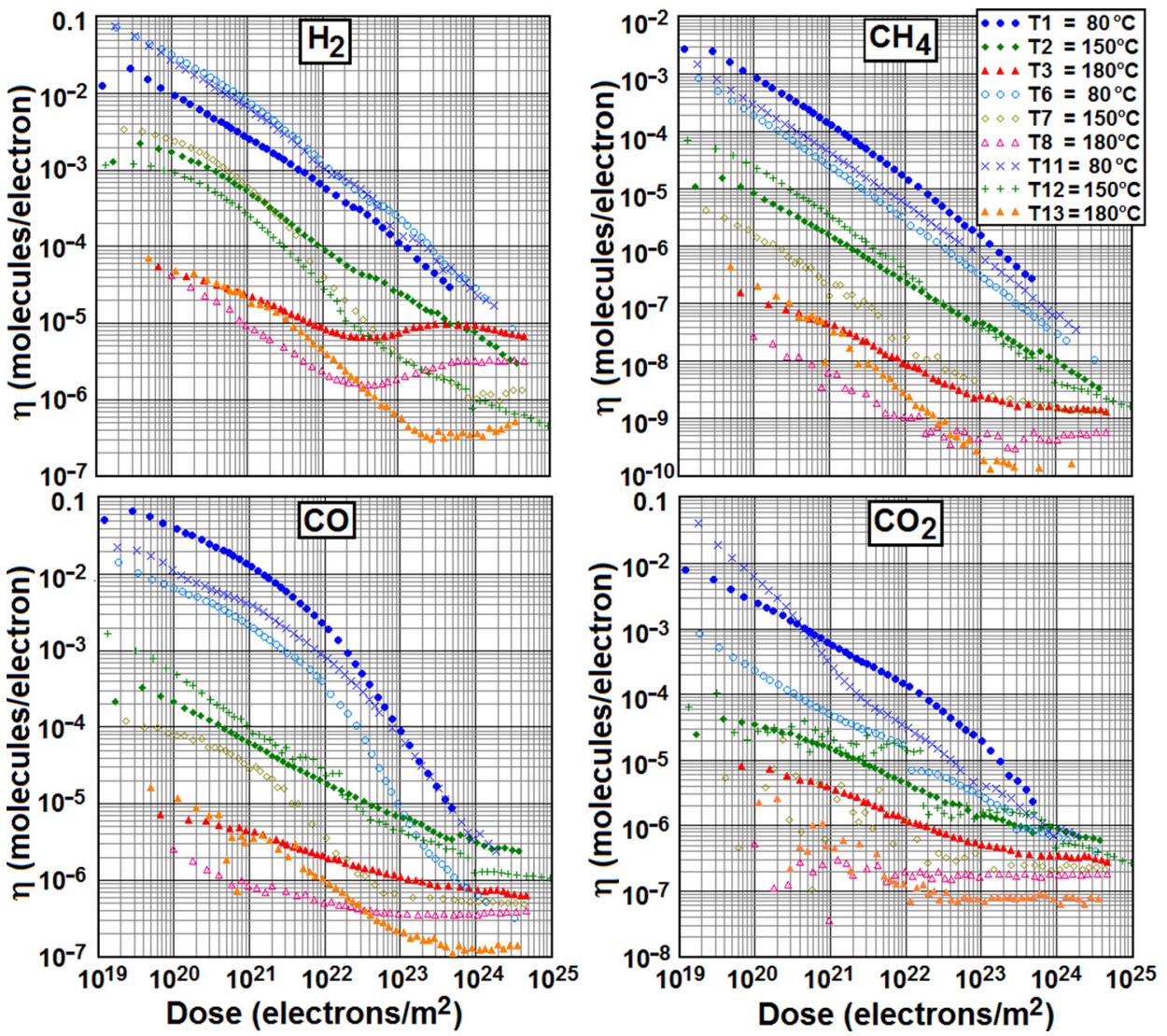

FIG. 4. (Color online) Comparison of ESD yields as a function of electron dose for samples activated at 80,150 , and $180{ }^{\circ} \mathrm{C}$.

Refs. 18 and 19. This leads to the conclusion that the most suitable activation temperature should be between 150 and $180^{\circ} \mathrm{C}$

For the operation of particle accelerators, it is important to understand the behavior of the NEG coating after air venting occurs. Comparison of the results from runs 1, 6 , and 11 demonstrates that the memory effect of the dual film is negligible even after a short air vent, whereby the ESD yields are 3-5 orders of magnitude higher than those prior to air venting. As can be seen from Fig. 4, $\eta(D)$ graphs obtained

TABLE II. Comparison of the results for NEG films prepared at different conditions: columnar (col) and dense (den) (Ref. 18), columnar (col vf) and dense (den vf) on vacuum fired substrates (Ref. 19) and dual layer (dual).

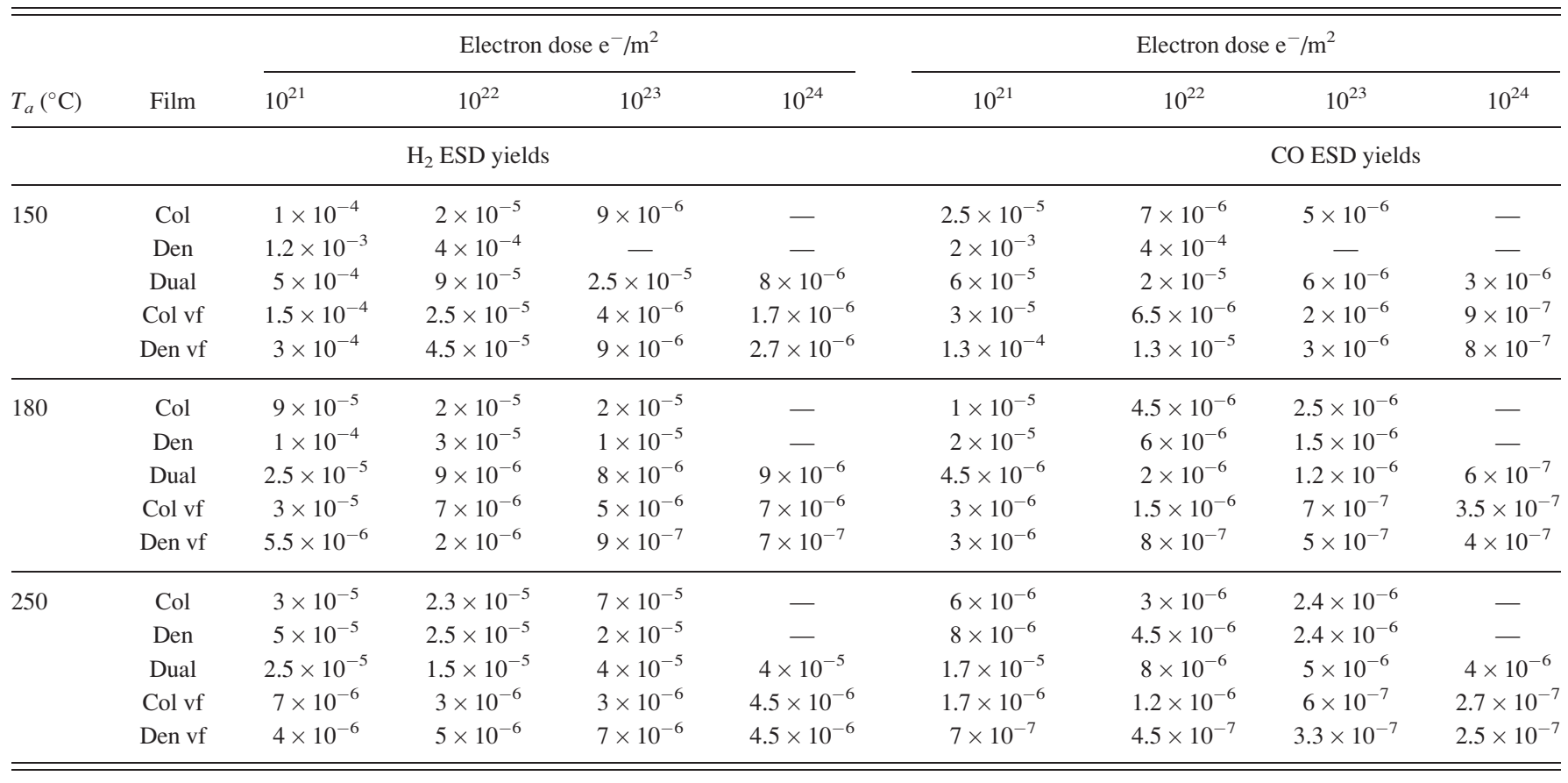



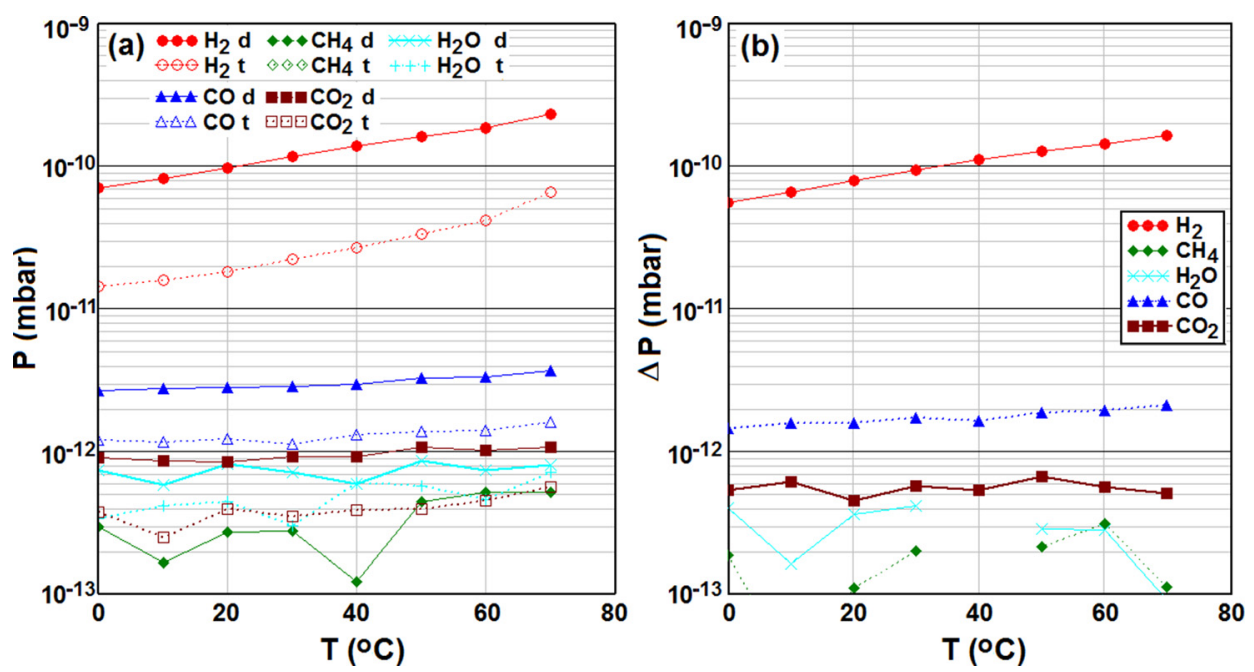

FIG. 5. (Color online) Pressure inside NEG-coated tube (top RGA) (a) with and without electron bombardment (indicated with d and t, respectively) and (b) their pressure difference $\Delta P$.

using the same activation temperatures are very similar. In addition, there is no degradation of the film properties.

During operation of the particle accelerator, minor vacuum accidents can occur. For example, the pressure inside the vacuum chamber can be increased to low or medium vacuum owing to an air leak or accidental gas injection. In these events, the NEG coating may be fully saturated even if the source of the accidental gas was mitigated without venting the vacuum system to air. A minor vacuum accident was simulated in run 10 , when the NEG coating was fully saturated with $\mathrm{CO}$ at $P=10^{-3}$ mbar for $10 \mathrm{~min}$ and then bombarded with electrons after a $2 \mathrm{~h}$ pumping with a turbomolecular pump. In this case, the ESD yields of $\mathrm{CH}_{4}$, $\mathrm{CO}$, and $\mathrm{CO}_{2}$ behaved very similar to those in run 7 (after activation to $\left.150^{\circ} \mathrm{C}\right)$, and the initial $\eta\left(\mathrm{H}_{2}\right)$ value was 30 times lower than that in run 7 (Fig. 3). This demonstrates that the NEG coating may not require reactivation after a minor vacuum accident owing to the so-called memory effect.

The behavior of the vacuum chambers after a minor accident was also simulated under different conditions in run 13, which induced a full saturation with $\mathrm{CO}$ and a 1-year long pumping followed by reactivation at $180^{\circ} \mathrm{C}$. While the long pumping duration did not affect the initial ESD yields compared to those in runs 3 and 8 (also activated at $180^{\circ} \mathrm{C}$ ), at doses $D>3 \times 10^{22} \mathrm{e}^{-} / \mathrm{m}^{2}$, the ESD yields were found to be lower for all gases. Therefore, it is advisable to maintain NEG-coated vacuum chambers under vacuum even when the NEG coating has been saturated or not activated.

The effect of vacuum chamber temperatures between 0 and $70^{\circ} \mathrm{C}$ upon the pressure inside a vacuum chamber with and without electron bombardment is shown in Fig. 5. Both the pressures, without electron bombardment (defined by thermal outgassing and indicated with " $t$ " in Fig. 5) and with electron bombardment (dynamic pressure indicated with "d"), clearly increase with temperature for $\mathrm{H}_{2}$ and $\mathrm{CO}$, and their pressure difference $\Delta P$ (defined by ESD) varies by factors of 3 and 1.5 for $\mathrm{H}_{2}$ and $\mathrm{CO}$, respectively. For the other gases, the signal is within a noise level to make any reasonable conclusions. These results are complementary to those reported in Ref. 22, where the equilibrium $\mathrm{H}_{2}$ pressure was studied in a vacuum chamber with a varying temperature between 100 and $250^{\circ} \mathrm{C}$.

\section{CONCLUSIONS}

A dual-layer NEG coating consisting of a $0.5-\mu \mathrm{m}$-thick dense layer covered by a $1-\mu \mathrm{m}$ thick columnar layer was evaluated for the first time. It was demonstrated that this dual-layer coating combined the advantages of the reduced ESD yields of a dense layer and the pumping properties of a columnar layer. Exposure to air required reactivation of the NEG coating, though reactivation was not required after minor vacuum incidents that led to full saturation. The NEG coating possessed negligible sensitivity to vacuum chamber temperatures between 0 and $70^{\circ} \mathrm{C}$, whereby $\Delta P$ varied by factors of 3 and 1.5 for $\mathrm{H}_{2}$ and $\mathrm{CO}$, respectively.

\section{ACKNOWLEDGMENTS}

This work was conducted under the aegis of the Science and Technology Facility Council (STFC). The authors would also like to acknowledge the support provided by the EuroCirCol collaboration (European Union's H2020 Framework Program under Grant Agreement No. 654305).

${ }^{1}$ C. Benvenuti, "Non-evaporable getters; from pumping strips to thin film coatings," in Proceedings of the EPAC-1998, Stockholm, Sweden (1998), p. 200.

${ }^{2}$ C. Benvenuti, P. Chiggiato, F. Cicoira, and V. Ruzinov, Vacuum 50, 57 (1998).

${ }^{3}$ C. Benvenuti, A. Escudeiro Santana, and V. Ruzinov, Vacuum 60, 279 (2001).

${ }^{4}$ R. Kersevan, "Performance of a narrow-gap, NEG-coated, extruded aluminium vacuum chamber at the ESRF," in Proceedings of the EPAC2000, Vienna, Austria (2000), p. 2291.

${ }^{5}$ C. Herbeaux, N. Bèchu, A. Conte, P. Manini, A. Bonucci, and S. Raimondi, "NEG coated chambers at SOLEIL: Technological issues and 
experimental results," in Proceedings of the EPAC08, Genoa, Italy (2008), p. 3711.

${ }^{6}$ G. Bregliozzi, T. Porcelli, G. Lanza, V. Baglin, and J. M. Jimenez, "Evaluation of the NEG coating saturation level after 3 years of ILHC beam operation," in Proceedings of the IPAC'13, Shanghai, China (2013), p. 3397.

${ }^{7}$ S. Calatroni, P. Chiggiato, P. Costa Pinto, M. Taborelli, M. Grabski, J. Ahlbäck, E. Al-Dmour, and P. Fernandes Tavares, "NEG thin film coating development for the MAX IV vacuum system," in Proceedings of the IPAC'13, Shanghai, China (2013), p. 3385.

${ }^{8}$ C. Benvenuti, P. Chiggiato, P. Costa Pinto, A. Escudeiro Santana, T. Hedley, A. Mongelluzzo, V. Ruzinov, and I. Wevers, Vacuum 60, 57 (2001).

${ }^{9}$ V. V. Anashin, I. R. Collins, R. V. Dostovalov, N. V. Fedorov, A. A. Krasnov, O. B. Malyshev, and V. L. Ruzinov, Vacuum 75, 155 (2004).

${ }^{10} \mathrm{P}$. Chiggiato and R. Kersevan, Vacuum 60, 67 (2001).

${ }^{11}$ F. Le Pimpec, O. Grobner, and J. M. Laurent, Nucl. Instrum. Methods Phys. Res., B 194, 434 (2002).

${ }^{12}$ O. B. Malyshev, "Vacuum systems for the ILC damping rings," EuroTeV Report-2006-094, 2006.
${ }^{13}$ O. B. Malyshev, V. V. Anashin, I. R. Collins, R. V. Dostovalov, N. V. Fedorov, A. A. Krasnov, and V. L. Ruzinov, J. Vac. Sci. Technol., A 23, 570 (2005).

${ }^{14}$ O. B. Malyshev, Vacuum 86, 1669 (2012).

${ }^{15}$ C. Benvenuti, P. Chiggiato, P. Costa Pinto, A. Prodromides, and V. Ruzinov, Vacuum 71, 307 (2003).

${ }^{16}$ O. B. Malyshev, R. Valizadeh, J. S. Colligon, A. Hannah, K. J. Middleman, S. Patel, and V. M. Vishnyakov, J. Vac. Sci. Technol., A 27, 521 (2009).

${ }^{17}$ O. B. Malyshev, R. Valizadeh, and A. Hannah, Vacuum 100, 26 (2014).

${ }^{18}$ O. B. Malyshev, R. Valizadeh, R. M. A. Jones, and A. Hannah, Vacuum 86, 2035 (2012).

${ }^{19}$ O. B. Malyshev, R. Valizadeh, B. T. Hogan, and A. Hannah, J. Vac. Sci. Technol., A 32, 061601 (2014).

${ }^{20}$ O. B. Malyshev, A. P. Smith, R. Valizadeh, and A. Hannah, J. Vac. Sci. Technol., A 28, 1215 (2010).

${ }^{21}$ O. B. Malyshev and K. J. Middleman, Vacuum 83, 976 (2009).

${ }^{22} \mathrm{~A}$. Rossi, " $\mathrm{H}_{2}$ equivalent pressure with NEG coated vacuum chamber as a function of temperature and $\mathrm{H}_{2}$ concentration," in Proceedings of the EPAC-2006, Edinburgh, Scotland (2006), p. 1444. 